\title{
INFORMAÇÕES ACERCA DE MARCADORES MOLECULARES UNIPARENTAIS: DNA MITOCONDRIAL E CROMOSSOMO Y
}

\author{
Information Concerning Molecular Markers Uniparentals: \\ Mitochondrial DNA And Chromosome Y
}

Ítalo Moraes de Magalhães ${ }^{1}$

Daniela de Melo e Silva ${ }^{2}$

\section{Resumo}

O presente trabalho visa mostrar a importância do uso de STRs (Seqüências curtas de repetições em Tandem) como ferramentas de identificação individual, devido ao alto grau de polimorfismo, e por formar um perfil genético único para cada indivíduo, exceto em gêmeos monozigóticos. Por isso, se tornou um importante marcador nos estudos de vínculo genético, identificação criminal de suspeitos e estudos de filogenia. Por causa do excelente nível de discriminação e ótima reprodutibilidade, estes marcadores fazem parte de várias pesquisas em todo o mundo, resultando em um rico banco de dados sobre as diferentes populações existentes. Serão destacados os marcadores STR do cromossomo Y que juntamente com o DNA mitocondrial permitem estudos estratégicos em diferentes áreas da genética. A Ciência Forense tem sido beneficiada cada vez mais com a Tecnologia do DNA, permitindo, por meio da tipagem ou "impressão digital" do DNA, solucionar questões cruciais, que até então, sem esse artifício, seriam praticamente impossíveis de alcançar um consenso. A variação genética detectada pelo mtDNA e Cromossomo Y, em comparação com os autossomos, são mais suscetíveis aos efeitos do acaso da genética, levando a diferenciações de agrupamentos geográficos e a diferenciação entre grupos de mtDNA e linhagens do Cromossomo Y.

Palavras-chave: STRs; Ciência Forense; Microssatélites; mtDNA; Cromossomo Y.

1 Ítalo Moraes de Magalhães, "Biomédico" pela Universidade Católica de Goiás (UCG) e Discente do Programa de Especialização em Genética, Universidade Católica de Goiás, Goiânia-Goiás. Endereço para correspondência: Rua 55, n 779, ap. 11, Centro. CEP: 74055-150. Goiânia-GO, Brasil. E-mail: italo.moraes@uol.com.br.

2 Daniela de Melo e Silva, Docente do Programa de Especialização em Genética, Universidade Católica de Goiás, Goiânia-Goiás; Núcleo de Pesquisas Replicon, Departamento de Biologia, Universidade Católica de Goiás, Goiânia-Goiás, Brasil. 


\section{Abstract}

The present work showed the importance of the use of STRs (Sequence in Tandem Repeats) as tools for individual identification, and the highly polymorphism is capable to form an only genetic profile for each individual, except for monozygotic twins. Therefore, it turned an important marker in the studies of genetic profiles, criminal suspect's identification and phylogeny studies. Because of the excellent level of discrimination and the great reproducibility of these markers, STR are part of several researches in all over the world, resulting in a rich database of the different populations. Y STR and Mitochondrial DNA allowed strategical studies in different areas of genetics. The Forensic Science has been benefited more and more with the DNA Technology, allowing, through of DNA typing or DNA "fingerprint" to solve crucial problems, that without this artifice, they would be practically irresolvable. The genetic variation, detected by mtDNA and Chromosome $\mathrm{Y}$, is more susceptible to stochastic effects, formiming geographical groups and distinct lineages of mtDNA and Chromosome Y haplogroups.

Keywords: STRs; Forensic Science; Microsatellites; mtDNA; Chromosome Y.

\section{Introdução}

Na década de 80, os primeiros dados foram publicados sobre o uso de fragmentos de DNA chamados VNTRs (Número variável de repetições em Tandem), ou seja, fragmentos com repetições de 9 a 80 pares de base, que seriam utilizados como ferramentas de identificação individual (1). Os VNTRs são regiões do DNA altamente polimórficas, que quando estudadas em conjunto, podem formar um perfil genético único para um indivíduo (2).

No final dos anos oitenta, novas seqüências com características similares aos VNTRs, porém de tamanho menor e com cerne de repetição mais curto, foram descobertas (3). Estas seqüências foram denominadas de microssatélites ou STR (Seqüências curtas de repetições em Tandem), ou seja, seqüências de DNA mais utilizadas com a finalidade de identificação individual com um nível de discriminação excelente e ótima reprodutibilidade $(4,5)$. Em relação ao uso dos microssatélites, nos estudos de vínculo genético, algumas vantagens em relação às VNTRs podem ser citadas, incluindo a rapidez e facilidade técni$\mathrm{ca}$, resultados mais objetivos, possibilidade de estudar um maior número de loci e o poder discriminatório ao nível de um par de base (6).

Os marcadores STRs são seqüências de DNA dispersas por todo o genoma, que contêm unidades repetidas de 2 a 6 pares de base, podendo ser facilmente amplificadas por PCR (Reação em Cadeia da Polimerase) (7). Por serem altamente polimórficos, são marcadores importantes em estudos de vínculo genético (4), identificação criminal de suspeitos, estudos de filogenia, dentre outros $(8,9)$.
A maior parte dos estudos conduzidos em relação aos microssatélites tem revelado que suas freqüências genotípicas apresentam-se em equilíbrio de Hardy-Weinberg $(10,11)$, uma característica importante para que o marcador possa ser útil nos estudos populacionais (12).

Estes marcadores fazem parte de várias pesquisas por todo o mundo e os resultados geram um rico banco de dados sobre populações de diferentes continentes. Suas seqüências são amplamente conhecidas e disponíveis em alguns bancos de dados na Internet como pode ser encontrado no National Institute of Standards and Technology - NIST. Nesta revisão serão destacados os marcadores STR do cromossomo Y que juntamente com o DNA mitocondrial permitem estudos estratégicos em diferentes áreas da genética.

O DNA mitocondrial humano (mtDNA) é uma molécula circular de fita dupla apresentando 16,569 pares de base com sua seqüência inteira conhecida. Funcionalmente é dividido em duas regiões denominadas como: controle e codificante. O genoma mitocondrial codifica 37 genes; 22 RNA transportadores, 2 RNAs ribossomais e 13 proteínas envolvidas no processo de fosforilação oxidativa. Tal genoma apresenta herança materna sem recombinação. Uma outra característica importante que diferencia o DNA mitocondrial do DNA nuclear é a taxa de mutação, sendo que a taxa de mutação do DNA mitocondrial é de dez a cem vezes maior do que a taxa do DNA nuclear, devido à falta de histonas a um sistema de reparo menos efetivo. A taxa de mutação observada no genoma mitocondrial é mais alta na região controle do que na região codificante (13).

A Ciência Forense tem sido beneficiada cada vez mais com a Tecnologia do DNA tanto 
pela genotipagem ou "impressão digital" do DNA, solucionando questões cruciais. Assim, nenhuma outra área tem se utilizado mais desse tipo de ferramenta do que a Biologia Molecular, isto porque, há mais de 15 anos, o método de análise de tipagem do DNA está sendo utilizado no mundo inteiro para solucionar casos de identificação em crimes violentos, crimes menores, atos de terrorismo, desaparecimento de pessoas e desastres em massa (14).

O genoma humano é representado por apenas 2 seguimentos haplóides, mtDNA (herdado maternalmente) e a região que não sofre recombinação do cromossomo Y (herdado paternalmente), desde que estes sejam transmitidos uniparentalmente, sem arranjo em cada geração por meio da recombinação do processo. Devido à recombinação de escape, esses 2 sistemas haplóides são herdados de um único loci que se modifica apenas por meio da mutação acumulada pelo tempo, o que permite a preservação de um simples registro da história genética. Em populações diferentes, especialmente as pequenas, a variação genética detectada pelo mtDNA e pelo Cromossomo Y, em comparação com os autossomos, são mais suscetíveis aos efeitos do acaso levando à aceleração do agrupamento geográfico e a diferenciação entre grupos de mtDNA e linhagens do Cromossomo Y. A mutação que ocorre no mtDNA a qual provém uma abundância de polimorfismo e um amplo número de alogrupos maternos que são maiores do que os paternos. A estrutura geral genealógica paterna e materna são indicativos da origem comum de quase todos os africanos o que evidencia que não se pode menosprezar os diferentes graus de mutação e a abundância de polimorfismo (13).

Outra ferramenta importante, na maioria dos laboratórios que trabalham com genética forense é a análise dos STRs do Cromossomo Y (YSTR), pois da mesma forma que o mtDNA, os halótipos Y-STR representam a informação de uma linhagem não recombinada que pode ser compartilhada por vários indivíduos. Todavia, durante a última década, a utilidade do Cromossomo Y tem sido reconhecida nos casos de deficiência de paternidade com descendência masculina e nos casos de genética forense, cujas análises autossômicas dos STRs falham em dar conclusões claras, isto porque, em uma larga proporção de machos e fêmeas, o perfil do macho só pode ser detectado pela análise dos marcadores do Cromossomo Y (15). O estudo do Cromossomo Y, por meio dos testes de DNA masculinos, possibilitará a descoberta da linhagem paterna, assim sendo, YSTRs podem ser utilizados em testes de identificação humana, incluindo análises forenses que evidenciem abuso sexual; condução de investigações de pessoas desaparecidas; testes de paternidade deficientes; localizar questões históricas; suplementando questões genealógicas. O estudo do Cromossomo Y adicionado a outros marcadores tem sido utilizado para investigar as causas genéticas da infertilidade masculina. Outro fator que tem contribuído para o estímulo de pesquisas dos marcadores do Cromossomo Y é o desejo que o ser humano possui de entender a sua evolução e migração (16).

Em síntese, o campo forense, mesmo com os benefícios que a Tecnologia do DNA vem lhe proporcionando, tem se deparado com um constrangimento no emprego desta, porque o seu uso não é considerado um procedimento rotineiro. Tal dificuldade se deve à disciplina científica chamada "Lei", que tem como uma de suas funções revisar a tecnologia. Portanto, mesmo que a finalidade seja a mesma, a do descobrimento da verdade, ressalta-se, aqui, que o modo como esse fato será obtido não ocorre da mesma forma para a ciência e a lei, porque, no caso da primeira, ela continuará insistentemente questionando convicções e descobertas. Todavia, metodologias de tipagem do DNA estarão continuamente sujeitas a exames minuciosos tanto da ciência quanto da lei, mesmo que muitos destes métodos tenham sido dedicados para tipagem nuclear do DNA (14).

Desta forma, este trabalho de revisão teve como objetivo caracterizar os principais marcadores STR do Cromossomo Y (YSTR) utilizados em estudos forenses e genéticos- evolutivos.

\section{Ma terial e Método}

Para evidenciar os principais marcadores localizados no Cromossomo Y, que são utilizados em estudos forenses e genético-evolutivos, foi acessado o site do NIST (http://www.cstl.nist.gov/ biotech/strbase/). Tal site contém um banco de dados genéticos que caracteriza todos os marcadores STR, dos cromossomos autossômicos e sexuais, evidenciando o cerne de repetição, as- 
sim como o número de repetição em tandem e o número de acesso no GenBank.

Foram selecionados 76 marcadores STR do Cromossomo Y que são utilizados em laboratórios de biologia molecular. Destes 76 marcadores foram selecionados 2 para que pudéssemos evidenciar o tamanho dos fragmentos amplificados e o número de repetições alélicas.

\section{Resultados}

A seguir, a tabela abaixo caracteriza os 76 marcadores de YSTR selecionados no NIST.

\section{TABELA 1 - Lista resumida dos marcadores STRs do Cromossomo Y}

\begin{tabular}{|c|c|c|c|}
\hline Nome do Marcador & $\begin{array}{l}\text { Número de } \\
\text { Repetições }\end{array}$ & $\begin{array}{l}\text { Seqüência de } \\
\text { Repetição }\end{array}$ & $\begin{array}{l}\text { N.o de Acessos } \\
\text { no GenBank }\end{array}$ \\
\hline DYS19 & $10-19$ & TAGA & AC017019 \\
\hline DYS385 a/b & $7-28$ & GAAA & AC022486 \\
\hline \multirow[t]{2}{*}{ DYS389 I DYS389 II } & DYS389I: 9-17 & (TCTG) (TCTA) & $\mathrm{AC} 004617$ \\
\hline & DYS389II:24-34 & (TCTG) (TCTA) & \\
\hline DYS390 & $17-28$ & (TCTA) (TCTG) & AC011289 \\
\hline DYS391 & $6-14$ & TCTA & AC011302 \\
\hline DYS392 & $6-17$ & TAT & $\mathrm{AC} 011745$ \\
\hline DYS393 & $9-17$ & AGAT & AC006152 \\
\hline YCAII a/b & $11-25$ & $\mathrm{CA}$ & AC015978 \\
\hline DYS388 & $10-18$ & ATT & AC004810 \\
\hline DYS425 & $10-14$ & TGT & AC095380 \\
\hline DYS426 & $10-12$ & GTT & AC007034 \\
\hline DYS434 & $9-12$ & TAAT (CTAT) & AC002992 \\
\hline DYS435 & $9-13$ & TGGA & AC002992 \\
\hline DYS436 & $9-15$ & GTT & AC005820 \\
\hline DYS437 & $13-17$ & TCTA & AC002992 \\
\hline DYS438 & $6-14$ & TTTTC & AC002531 \\
\hline DYS439 & $9-14$ & AGAT & AC002992 \\
\hline DYS441 & $12-18$ & CСTT & AC004474 \\
\hline DYS442 & $10-14$ & TATC & AC004810 \\
\hline DYS443 & $12-17$ & TTCC & AC007274 \\
\hline DYS444 & $11-15$ & TAGA & AC007043 \\
\hline DYS445 & $10-13$ & TTTA & AC009233 \\
\hline DYS446 & $10-18$ & ТСТСТ & AC006152 \\
\hline DYS447 & $22-29$ & TAAWA & AC005820 \\
\hline DYS448 & $20-26$ & AGAGAT & AC025227 \\
\hline DYS449 & $26-36$ & TTTC & AC051663 \\
\hline DYS450 & $8-11$ & TTTTA & AC051663 \\
\hline DYS452 & $27-33$ & YATAC & AC010137 \\
\hline DYS453 & $9-13$ & AAAT & AC006157 \\
\hline DYS454 & $10-12$ & AAAT & AC025731 \\
\hline DYS455 & $8-12$ & AAAT & AC012068 \\
\hline DYS456 & $13-18$ & AGAT & AC010106 \\
\hline DYS458 & $13-20$ & GAAA & AC010902 \\
\hline DYS459 a/b & $7-10$ & TAAA & AC010682 \\
\hline DYS460 (A7.1) & $7-12$ & ATAG & AC009235 \\
\hline
\end{tabular}


continuação...

\begin{tabular}{|c|c|c|c|}
\hline Nome do Marcador & $\begin{array}{l}\text { Número de } \\
\text { Repetições }\end{array}$ & $\begin{array}{l}\text { Seqüência de } \\
\text { Repetição }\end{array}$ & $\begin{array}{l}\text { N.o de Acessos } \\
\text { no GenBank }\end{array}$ \\
\hline DYS461 (A7.2) & 8-14 & (TAGA) CAGA & AC009235 \\
\hline DYS462 & $8-14$ & TATG & AC007244 \\
\hline DYS463 & $18-27$ & $\mathrm{AARGG}$ & AC007275 \\
\hline DYS $464 \mathrm{a} / \mathrm{b} / \mathrm{c} / \mathrm{d}$ & $11-20$ & ССТT & X17354 \\
\hline DYS481 & $20-30$ & CTT & - \\
\hline DYS485 & $10-18$ & TTA & - \\
\hline DYS490 & - & TTA & AC019058 \\
\hline DYS495 & $12-18$ & AAT & AC004474 \\
\hline DYS497 & $13-16$ & TAT & - \\
\hline DYS504 & - & - & - \\
\hline DYS505 & $9-15$ & TCCT & AC012078 \\
\hline DYS508 & $8-15$ & TATC & AC006462 \\
\hline DYS520 & $18-26$ & ATAS & AC007275 \\
\hline DYS522 & $8-17$ & GATA & AC007247 \\
\hline DYS525 & & TAGA & AC010104 \\
\hline DYS531 & $11-13$ & AAAT & - \\
\hline DYS532 & $9-17$ & CTTT & AC016991 \\
\hline DYS533 & $9-14$ & ATCT & AC053516 \\
\hline DYS534 & $10-20$ & СTTT & AC053516 \\
\hline DYS540 & - & TTAT & AC010135 \\
\hline DYS549 & $10-14$ & AGAT & - \\
\hline DYS556 & - & AATA & AC011745 \\
\hline DYS557 & - & TTTC & AC007876 \\
\hline DYS565 & $9-14$ & TAAA & - \\
\hline DYS570 & $12-23$ & TTTC & AC012068 \\
\hline DYS572 & $8-12$ & AAAT & - \\
\hline DYS573 & $8-11$ & TTTA & - \\
\hline DYS575 & - & AAAT & AC007247 \\
\hline DYS576 & $13-21$ & AAAG & AC010104 \\
\hline DYS594 & - & TAAAA & AC010137 \\
\hline DYS607 & - & AAGG & - \\
\hline DYS612 & - & - & - \\
\hline DYS614 & - & - & - \\
\hline DYS626 & - & AAAG & - \\
\hline DYS632 & - & CATT & AC006371 \\
\hline DYS635 (C4) & $17-27$ & TSTA & AC004772 \\
\hline DYS641 & - & TAAA & AC018677 \\
\hline DYS643 & $7-15$ & CTTTT & AC007007 \\
\hline Y-GATA-H4 & $8-13(25-30)$ & TAGA & AC011751 \\
\hline Y-GATA-C4 & $20-25$ & TSTA & G42673 \\
\hline Y-GATA-A10 & $13-18$ & TAGA & AC011751 \\
\hline
\end{tabular}

Fonte: NIST. Summary List of Y Chromosome STR Lociand Available Fact Sheets. Disponível em: <http:// www.cstl.nist.gov/div831/strbase/ystr_fact.htm>. Acesso em: 12 jan. 2006. 


\section{Tabela 2 - Descrição do marcador DYS19}

\begin{tabular}{lll}
\hline $\begin{array}{l}\text { Número de Repetições } \\
\text { Alélicas }\end{array}$ & $\begin{array}{l}\text { Tamanhos dos Fragmentos } \\
\text { Amplificados }\end{array}$ & Cerne de repetição \\
\hline 8 & $178 \mathrm{bp}$ & \\
$10(\mathrm{~A})$ & $186 \mathrm{bp}$ & {$[\text { TAGA }]_{10}$} \\
$11(\mathrm{~B})$ & $190 \mathrm{bp}$ & {$[\text { TAGA }]_{11}$} \\
$12(\mathrm{C})$ & $194 \mathrm{bp}$ & {$[\mathrm{TAGA}]_{12}$} \\
$13(\mathrm{D})$ & $198 \mathrm{bp}$ & {$[\text { TAGA }]_{13}$} \\
$14(\mathrm{E})$ & $202 \mathrm{bp}$ & {$[\text { TAGA }]_{14}$} \\
$15(\mathrm{~F})$ & $206 \mathrm{bp}$ & {$[\text { TAGA }]_{15}$} \\
$16(\mathrm{G})$ & $210 \mathrm{bp}$ & {$[\text { TAGA }]_{16}$} \\
\hline
\end{tabular}

Fonte: NIST. DYS19. Disponível em: <http://www.cstl.nist.gov/div831/strbase/strbase/str_ dy19.htm >. Acesso em: 12 jan. 2006.

\section{TABELA 3 - Descrição do marcador DYS385 a/b}

\begin{tabular}{|c|c|c|c|c|}
\hline $\begin{array}{l}\text { Numero de } \\
\text { Repetições } \\
\text { Alélicas }\end{array}$ & $\begin{array}{l}\text { Tamanhos dos } \\
\text { Fragmentos } \\
\text { Amplificados }\end{array}$ & $\begin{array}{l}\text { Tamanhos dos } \\
\text { Fragmentos } \\
\text { Amplificados }\end{array}$ & $\begin{array}{l}\text { Tamanhos dos } \\
\text { Fragmentos } \\
\text { Amplificados }\end{array}$ & $\begin{array}{l}\text { Cerne de } \\
\text { repetição }\end{array}$ \\
\hline 7 & 352 & 241 & 242 & {$\left[\mathrm{GAAA}_{7}\right.$} \\
\hline 8 & 356 & 245 & 246 & GAAA $_{8}$ \\
\hline 9 & 360 & 249 & 250 & {$[\mathrm{GAAA}]_{9}$} \\
\hline 10 & 364 & 253 & 254 & {$[\mathrm{GAAA}]_{10}$} \\
\hline 11 & 368 & 257 & 258 & {$[\mathrm{GAAA}]_{11}^{10}$} \\
\hline 12 & 372 & 261 & 262 & {$[\mathrm{GAAA}]_{12}$} \\
\hline 13 & 376 & 265 & 266 & {$[\mathrm{GAAA}]_{13}^{12}$} \\
\hline 14 & 380 & 269 & 270 & {$[\mathrm{GAAA}]_{14}$} \\
\hline 15 & 384 & 273 & 274 & {$[\mathrm{GAAA}]_{15}$} \\
\hline 16 & 388 & 277 & 278 & {$\left[\mathrm{GAAA}_{16}\right.$} \\
\hline 16.3 & 391 & 280 & 279 & {$[\mathrm{GAAA}]_{17}$} \\
\hline 17 & 392 & 281 & 282 & {$\left[\mathrm{GAAA}_{17}\right.$} \\
\hline 17.2 & 394 & 282 & 283 & {$\left[\mathrm{GAAA}_{1}[\mathrm{GA}]_{1}[\mathrm{GAAA}]_{16}\right.$} \\
\hline 17.3 & 395 & 283 & 284 & {$[\mathrm{GAAA}]_{18}$} \\
\hline 18 & 396 & 284 & 286 & {$[\mathrm{GAAA}]_{18}$} \\
\hline 19 & 400 & 288 & 290 & {$[\mathrm{GAAA}]_{19}^{18}$} \\
\hline 20 & 404 & 292 & 294 & {$[\mathrm{GAAA}]_{20}$} \\
\hline 21 & 408 & 296 & 298 & {$[\mathrm{GAAA}]_{21}^{20}$} \\
\hline 22 & 412 & 300 & 302 & {$[\mathrm{GAAA}]_{22}^{21}$} \\
\hline 23 & 416 & 304 & 306 & {$[\mathrm{GAAA}]_{23}^{22}$} \\
\hline 24 & 420 & 308 & 310 & desconhecido \\
\hline 28 & 436 & 324 & 326 & desconhecido \\
\hline
\end{tabular}

Fonte: NIST. DYS385 a/b. Disponível em: <http://www.cstl.nist.gov/div831/strbase/str_y385.htm>. Acesso em: 12 jan. 2006. 


\section{Discussão e Conclusão}

Atualmente, cerca de 220 STRs, que são potencialmente usados em genética forense, têm sido identificados no cromossomo Y humano. Para a maioria deles, dados relevantes no cerne de repetição e a capacidade discriminatória de tais marcadores ainda são questionáveis, portanto é prematuro recomendar qualquer um dos novos STRs para propósitos forenses (15).

Mais de um marcador YSTR pode ser examinado simultaneamente com a reação de PCR Multiplex, economizando tempo e esforços na tentativa de agrupar informações de outros marcadores genéticos. Primers bem desenhados e de alta qualidade são essenciais para obter sucesso nas reações de multiplex. O laboratório do U.S. National Institute of Standars and Technology (NIST) está envolvido desde 2000 no desenvolvimento de novos testes de YSTR e tem melhorado a padronização da informação dos marcadores do cromossomo Y. A PCR multiplex tem sido usada com sucesso para aumentar mais do que 20 diferentes produtos dos marcadores Y-STR (17).

Os trabalhos ligados à pesquisa Retrato Molecular do Brasil identificam 10 haplogrupos, sendo os dois mais freqüentes o haplogrupo 1 (54\%) e o haplogrupo 2 (18\%), que juntos alcançaram $72 \%$. Enquanto o primeiro tem sido encontrado em alta freqüência na Europa e não se faz presente na África e no Japão, o segundo está presente não somente na Europa, como também na África e na Ásia (18, 19, 20 apud 20).

O exemplo citado acima é somente uma aplicação dos estudos do Cromossomo Y para caracterizar a população brasileira em etnias. Entretanto, tais marcadores disponíveis nos bancos de dados genéticos podem ser utilizados em exames de vínculo genético, investigações periciais e estudos filogeográficos.

Sintetizando: bancos de dados de fontes seguras, como o NIST, facilitam a pesquisa de informações sobre os marcadores moleculares e o acesso às novas descobertas auxiliando milhares de pesquisadores que trabalham em prol da humanidade.

\section{Referências}

1. Jeffreys AJ, Wilson V, Thein SL. Individualspecific 'fingerprints' of human DNA. Nature 1985; 316:75-79.

2. Conselho Nacional de Pesquisa. A tecnologia do DNA na ciência forense. Ribeirão Preto: FUNPEC-RP; 1999.

3. Weber JL, May PE. Abundant class of human DNA polymorphisms which can be typed using the polymerase chain reaction. Am J Hum Genet 1989; 44(3):388-96.

4. Dawid AP, Mortera J, Pascali VL. Non-fatherhood or mutation? A probabilistic approach to parental exclusion in paternity testing. Forensic Sci Int 2001; 124:55-61.

5. Lee HS, Lee JW, Han GR, Hwang JJ. Motherless case in paternity testing. Forensic Sci Int 2000; 114:57-65.

6. Henke J, Henke L. Mutation rate in human microsatellites. Am J Hum Genet 1999; 64: 1473.

7. Ruitberg CM, Reeder DJ, Butler JM. New primer sets for Y chromosome and CODIS STR loci. Poster presented at the Eleventh. International Symposium on Human Identification Biloxi, MS; 2000 .

8. Toth A, Rabitsch KP, Galoya M, Schleiffer A, Buonomo SB, Nasmyth K.. Functional genomics identifies monopolin: a kinetochore protein required for segregation of homologs during meiosis I. Cell 2000; 103:1155-1168.

9. Agrawal S.; Khan F. Reconstructing recent human phylogenies with forensic STR loci: Astatistical approach. BMC Genetics 2005; 6:47.

10. Edwards A, Civitello A, Hammond HA, Caskey, CT. DNA typing and genetic mapping with trimeric and tetrameric tandem repeats. Am J Hum Genet 1991; 49:746-56.

11. Santos FR, Tyler-Smith C. Reading the human Y chromosome: the emerging DNA markers and human genetic history. Braz J Genet 1996; 19:665-670. 
12. Grattapaglia D, Sederoff R. Genetic linkage maps of Eucalyptus grandis and E: urophylla using a pseudo-testcross mapping strategy and RAPD markers. Genetics 1994; 137:1121-1137.

13. Peric M., Lauc LB, Klaric IM, Janicijevic B, Rudan P. Review of Croating Genetic Heritage as Revealed by Mitochondrial DNA and Y Chromosomal Lineages. Croat Med J 2005; 46(4):502-513.

14. Budowle B, Allard MW, Wilson MR, Chacraborty R. Forensics and Mitochondrial DNA: aplications, debates and foundations. Annu Rev Genomics Hum Genet 2003; 4:119-141.

15. Gusmão L, Butler JM, Carracedo A, Gill P, Kayser M, Mayr WR, et al. DNA commission of the international society of forensic genetics (ISFG): na update of the recommendations on the use of Y-STRs in forensic analysis. Sci Forensic interno 2006; 157:187-97.

16. National Institute of Standards and Technology (NIST). Recent Developments in Y-Short tandem repeat and Y-Single nucleotide polymorphism analysis. Forensic Science Review 2003; 15(2):91-111.

Institute of Standards and Technology NIST. Short Tandem Repeat DNA Internet DataBase 2006. Disponível em: http:// www.cstl.nist.gov/biotech/strbase/.
. Summary List of Y Chromosome STR Loci and Available Fact Shetts 2006. Disponível em: http://www.cstl.nist.gov/div831/strbase/ ystr_fact.htm.

17. Butler, J. M. Recent developments in Y-short tandem repeat and $\mathrm{Y}$-single nucleotide polymorphism analysis. Forensic Science Review 2003; 15(2):91-111.

18. Alves-Silva J, Santos MS, Guimarães PEM, Ferreira ACS, Bandelt HJ, Pena SDJ, Prado VF. The ancestry of Brazilian mtDNA lineages. American Journal of Human Genetics 2000; 67: 444-461.

19. Carvalho-Silva DR, Santos FR, Rocha J, Pena SDJ. The phylogeography of Brazilian Ychromosome lineages. American Journal of Human Genetics 2001; 68:281-286.

20. Pena SDJ, Carvalho-Silva DR, Alves-Silva J, Prado VF, Santos FR. 2000. Retrato molecular do Brasil. Ciência Hoje 2000; 159:16-25.

21. Santos RV, Maio OMC. Qual "retrato do Brasil"? Raça, biologia, identidades e política na era da genômica. Rio de Janeiro, RJ: Mana 2004; 10(1):61-95.

Recebido em / Received: February 16, 2006. Aceito em / Accepted: March 20, 2006. 